\title{
Localized and delocalized surface-plasmon-mediated light tunneling through monolayer hexagonal-close-packed metallic nanoshells
}

\author{
Chaojun Tang, Zhenlin Wang, * Weiyi Zhang, Shining Zhu, and Naiben Ming \\ Department of Physics, National Laboratory of Solid State Microstructure, Nanjing University, Nanjing 210093, China
}

Gang Sun

Beijing National Laboratory for Condensed Matter Physics, Institute of Physics, Chinese Academy of Sciences, Beijing 100080, China

Ping Sheng

Department of Physics, Hong Kong University of Science and Technology, Clear Water Bay, Kowloon, Hong Kong, China

(Received 3 March 2009; revised manuscript received 14 September 2009; published 5 October 2009)

\begin{abstract}
We studied theoretically light transmission through a monolayer of hexagonal-close-packed nanoparticles consisting of a metallic shell and a dielectric core. We found that light can transmit through the dense particle assemblies via excitation of a variety of surface-plasmons (SPs). Localized SPs confined within metal nanoshells can mediate a narrow-band dispersionless transmission resonance (TR). Wide-band TRs were also observed as a result of strong near-field interparticle SP couplings, forming hybrid modes that are either localized at the nanogaps between adjacent particles or confined in the lattice pores, or distributed across the structure, each with distinct dispersion characteristics. Optical tuning strategies of these TRs are also elucidated that can allow for observation of SP anticrossing effects.
\end{abstract}

DOI: 10.1103/PhysRevB.80.165401 PACS number(s): 73.20.Mf, 42.25.Bs, 78.67.Bf, 78.66.Bz

In the past decade, metal films perforated with a periodic array of subwavelength holes or slits attract much interest after the report of enhanced optical transmittance (EOT) by Ebbesen et al. ${ }^{1}$ The EOT phenomenon is generally attributed to propagating surface-plasmon polaritons (SPPs), ${ }^{2}$ which are excited by the incident light on the input side of the metal film, then evanescently tunnel to the exit side through the building up of strong electromagnetic fields above the apertures, and are finally re-emitted into optical far field. The SPP waves are Bragg scattered by the periodic apertures and thus are referred to as Bragg-type SPP modes. Although other theoretical models are also proposed, ${ }^{3}$ many efforts have been made to study whether localized resonance modes in apertures can also bring EOT which should be independent of periodicity. ${ }^{4}$

Individual metallic nanoparticles can support localized surface-plasmons (SPs), whose resonance frequency depends on the particle size, shape, and composition, and also on the surrounding medium. ${ }^{5}$ At localized SP resonances, the huge electric field enhancement established on particle surface leads to a wealth of optical properties useful in surface enhanced Raman scattering, ${ }^{6}$ optical antennas, ${ }^{7}$ and optical tweezers. ${ }^{8}$ For regularly spaced metal nanoparticles, the interactions of particle SP resonances can result in interesting collective optical properties. Lamprecht et al. ${ }^{9}$ and Hicks et al. ${ }^{10}$ have shown that the lineshape of these localized SPs can be controlled via coherent dipole far-field interactions. Recently extremely narrow plasmon resonances in one- and two-dimensional (2D) arrays of metal nanoparticles have been predicted ${ }^{11-13}$ and observed experimentally, ${ }^{14,15}$ which are revealed to be due to a diffraction coupling of localized SPs with a diffracted grazing wave at a Wood anomaly. ${ }^{14}$ With these great efforts, a clear physical picture has been established about light interaction with two-dimensional (2D) metal nanoparticle arrays, where the excitation of di- pole plasmon mode and its far-field interaction are dominant. However, less is known about the optical properties of dense metal nanoparticle arrays, ${ }^{16}$ where higher-order SP modes and their near-field interactions could become extremely important for controlling the collective optical properties. ${ }^{17} \mathrm{Re}$ cently, near-field interparticle localized SPs coupling in chains of closely spaced metal nanoparticles has been explored to guide electromagnetic energy with a lateral confinement below light diffraction limit. ${ }^{18}$

Some metallic nanostructures can support both localized and delocalized SPs and therefore exhibit rich optical phenomena related to their couplings when they are excited simultaneously. For example, Coyle et al. ${ }^{19}$ and Kelf et al. ${ }^{20}$ successfully identified the presence of both localized Mie and delocalized Bragg plasmons on nanostructured metal surfaces comprised of periodically arranged truncated spherical voids, through measurements of angle- and orientationresolved optical reflectivity, and demonstrated their strong coupling thus producing bonding and antibonding mixed SPs by carefully controlling the sample thickness. ${ }^{20}$ Sun and Chan ${ }^{21}$ predicted frequency-selective enhanced absorption of light for a periodic lattice of dielectric spheres buried just beneath the surface of a metal substrate, by tuning localized Mie and delocalized Bragg plasmons into a strong coupling region.

In this paper, we study theoretically multiple light scatterings when light propagates through a 2D array of dense noble metal nanoshells with a dielectric core, arranged in a hexagonal-close-packed (HCP) lattice. We will show that light can transmit through the metallodielectric (MD) composite nanostructures with a high transmittance via a variety of SP excitations. We found that localized void plasmons, as an embodiment of single-particle Mie resonance, can mediate a narrow-band transmission resonance due to a high $Q$ quality factor of noble metallic nanoshells. The strong inter- 
particle interactions of SPs can form hybrid modes that can propagate across the MD nanostructures with Bragg-like dispersions, or modes that are highly localized near the gaps between adjacent nanoshells. In addition, the MD structures can support localized SPs that are mainly confined within the pores of the 2D lattice, which create a flat-dispersion transmission resonance under off-normal incidence. Furthermore, the anticrossing of the different SPs can lead to suppression of transmission. The distinct roles of these SPs and their interactions played in transmittance have been demonstrated clearly through the evaluation of transmission spectra as well as field distributions.

The transmission spectra and the field distributions are calculated with the electromagnetic wave layer-multiplescattering theory formalism of Stefanou-YannopapasModinos, ${ }^{22}$ which has been shown to be highly efficient and accurate in calculations of electromagnetic properties of spherical objects. In this approach, ${ }^{22}$ the electromagnetic field is first expanded into spherical harmonics about each spherical scatterer, and then the scattering properties of a periodical plane of scatterers are obtained by summation of all scattering events. Finally, the scattered wave field is transformed into a plane-wave representation. For pure dielectric structures, excellent numerical convergence can be achieved by including spherical waves with angular momentum index up to $L_{\max }=7$ in the local expansion of electromagnetic waves. ${ }^{23,24}$ Such a numerical convergence has also been tested for MD photonic band-gap crystals consisting of three-dimensional arrays of perfect metallic spheres in different lattices and excellent agreement between the band structure and the transmission code is obtained using $L_{\max }=7$ in the angular momentum expansions and $372 \mathrm{D}$ reciprocal lattice vectors (whose cut-off length $R_{\max }=22$, in units of the reciprocal of lattice period) in the plane-wave expansions. ${ }^{25}$ For the closely spaced metallic nanoshells discussed here, however, a value of $L_{\max }=7$ is not sufficient to obtain stable numerical results. In order to calculate precisely the scattering properties associated with multipole resonances of single MD spheres ${ }^{26}$ and the effect of strong near-field coupling within the dense array, we took the angular momentum cutoff $L_{\max }=19$ and used $1092 \mathrm{D}$ reciprocal lattice vectors $\left(R_{\max }=40\right)$ to ensure a relative accuracy of $10^{-4}$ for spherical metallic nanoparticles with separations of at least $1 \mathrm{~nm}$.

Figure 1(a) shows schematically the nanostructures to be studied. The coordinate is chosen such that the metallic nanoshells lie on the $x y$ plane, with its origin located at the center of one shell. The incident light direction is defined by two angles, incidence angle $\theta$ (the angle between $z$ axis and the wave-vector $k$ ) and azimuth angle $\varphi$ (the angle between $x$ axis and the wave-vector in-plane component $k_{x y}$ ). The composite spheres have a dielectric core of dispersionless dielectric constant $\varepsilon_{1}$ and a metallic coating nanoshell with the inner and outer radii denoted by $R_{1}$ and $R_{2}$, respectively. The MD structure has a lattice period of $a$. The relative permittivity of the metal is described by a Drude model: $\varepsilon_{2}=1$ $-\omega_{p}^{2} /\left[\omega\left(\omega+i \tau^{-1}\right)\right]$, where $\omega_{p}$ is the plasma frequency and $\tau$ is the relaxation time related to energy loss. The parameter is taken to be $\hbar \omega_{p}=9.2 \mathrm{eV}$, which corresponds to the plasma energy of bulk silver.

In order to focus on the essence of the underlying physics,
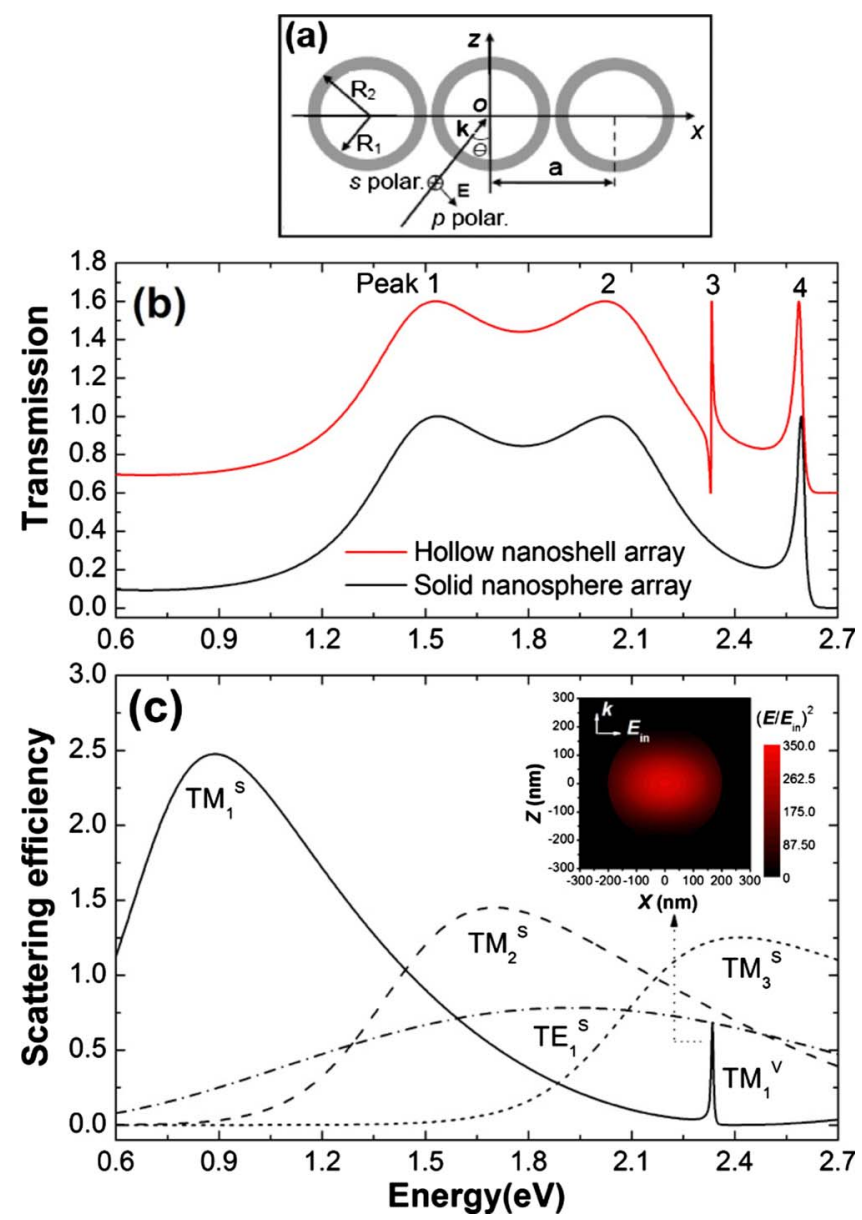

FIG. 1. (Color online) (a) Schematic of a 2D HCP array of metallic hollow nanoshells in air. (b) Normal-incidence transmission spectrum for a $2 \mathrm{D} \mathrm{HCP}$ array consisting of hollow $\mathrm{Ag}$ nanoshells with $R_{1}=200 \mathrm{~nm}, R_{2}=250 \mathrm{~nm}$, and $\varepsilon_{1}=1.0$, compared with that of solid $\mathrm{Ag}$ nanospheres with a diameter the same as the outer diameter of the nanoshells. The lattice period for both structures is $a=520 \mathrm{~nm}$. The spectrum of the nanoshell array is vertically offset by 0.6 for clarity. (c) Partial scattering efficiencies of a single hollow Ag nanoshell for several dominant transverse magnetic $\left(\mathrm{TM}_{l}^{V, S}\right)$ and transverse electric $\left(\mathrm{TE}_{l}^{V, S}\right)$ modes, where the subscript $l$ denotes the angular momentum index, and the superscripts $V$ and $S$ stand for voidlike and spherelike modes, respectively. The inset gives the normalized electric field intensity of a $\mathrm{TM}_{1}^{V}$ mode.

we begin our discussion by considering an ideal model in which the metal is lossless. The effect of absorption will be considered in the last part of the paper. Figure 1(b) presents the transmission spectrum of a 2D HCP array of hollow metallic nanoshells under normal illumination of light. The structural parameters are $R_{1}=200 \mathrm{~nm}, R_{2}=250 \mathrm{~nm}, a$ $=520 \mathrm{~nm}$, and $\varepsilon_{1}=1.0$. In Fig. 1(b), four conspicuous peaks with perfect transmission are observed which are located at $1.53,2.02,2.33$, and $2.59 \mathrm{eV}$, corresponding to wavelengths $810 \mathrm{~nm}\left(\lambda_{1}\right), 614 \mathrm{~nm}\left(\lambda_{2}\right), 532 \mathrm{~nm}\left(\lambda_{3}\right)$, and $479 \mathrm{~nm}\left(\lambda_{4}\right)$, respectively. The first three transmission resonances have a wavelength larger than the lattice period $a$. While the wavelength of the highest-energy resonance is slightly smaller than $a$, it is still much larger than the thinnest waist size of horn-shaped pores among adjacent shells in the lattice. It is 
noted that only a zero-order diffraction transmittance channel is open for the above MD structure in the spectral range discussed here. Thus, a metallic nanoshell array allows for light tunneling on a subwavelength scale, similar to 2D subwavelength nanohole arrays studied before., ${ }^{1,2}$

It is noted that the multiple transmission resonances are mediated by the excitation of SPs which will be shown to have different origins. We first discuss the SP mode that leads to the sharp transmission resonance at peak 3. For comparison, Fig. 1(b) also presents the transmission spectrum of a similar structure but composed of solid metallic spheres with an identical size as the nanoshell outer radius. We see that the solid-nanosphere assembly has almost the same spectral features as the nanoshell aggregate, except for the absence of peak 3 . This suggests that this narrow-band resonance is due to the excitation of a void mode of individual metallic nanoshells. To confirm this, partial scattering efficiencies of a single metallic nanoshell were calculated for the dominant transverse magnetic $\left(\mathrm{TM}_{l}^{V, S}\right)$ and transverse electric $\left(\mathrm{TE}_{l}^{V, S}\right)$ modes, by using the Mie theory ${ }^{27}$ and are plotted in Fig. 1(c). Here, the subscript $l$ denotes the angular momentum index, and the superscripts $V$ and $S$, respectively, stand for voidlike and spherelike modes of single metallic nanoshells as these modes are either confined within the inner space (for voidlike modes) or localized on the shell surface (for spherelike modes). The existence of these two types of SP modes makes spherical core/shell nanostructures especially attractive due to a unique structural tunability of the particle plasmons. ${ }^{28}$

It is clearly seen that the $\mathrm{TM}_{1}^{V}$ mode is responsible for peak 3 as they have nearly the same energy. A similar mechanism for light tunneling through thin metal films embedded with dielectric spheres on the surface has been proposed recently. ${ }^{29}$ In fact, the $\mathrm{TM}_{1}^{V}$ mode is a dipole plasmon resonance excited within the void of the nanoshells. The inset of Fig. 1(c) gives the electric field intensity distribution at such a resonance, from which we see that the fields are exclusively confined within the dielectric core. Such a strong localization makes the resonance frequency dependent only on the dielectric permittivity or the size of the core, but essentially independent of the structure periodicity. Since this mode is trapped and thus long lived when metal absorption is neglected, peak 3 has a very narrow bandwidth.

In order to reveal the physical origins for the other three transmission peaks in Fig. 1(b) which are basically the same for both MD nanostructures composed of either solid spheres or nanoshells of the same size, the electric field distributions around the nanoshell array were calculated for resonances at $\lambda_{1}, \lambda_{2}$, and $\lambda_{4}$. The corresponding numerical results are plotted in Fig. 2. At resonance $\lambda_{1}$, the fields are seen to be concentrated in the nanogaps between adjacent spheres along the $x$ direction (the polarization direction of incident light), known as hot spot. ${ }^{30}$ The resonance at $\lambda_{2}$, with its field distributions shown in Figs. 2(c) and 2(d), has a similar field pattern as the resonance at $\lambda_{1}$, except that the four spots on the sphere surface near the hot spot become distinguishable in intensity. Based on the field distributions and on the evolution of transmission spectra obtained by increasing the angular momentum cutoff $L_{\max }$ from 1 to higher values (not shown here), we conclude that peaks 1 and 2 are mainly
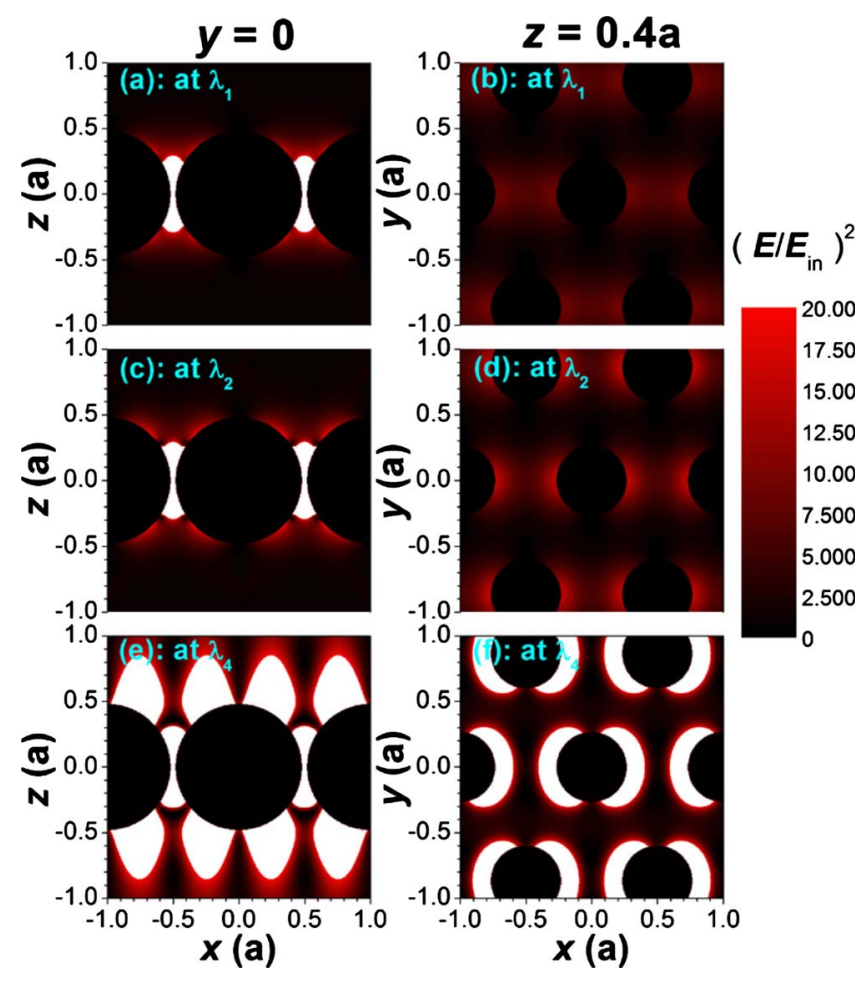

FIG. 2. (Color online) Normalized electric field intensity $\left(E / E_{\text {in }}\right)^{2}$ on the planes of $y=0$ (left column) and $z=0.4 a$ (right column) for peak 1 (top panels), peak 2 (middle panels), and peak 4 (bottom panels). The maximum in the color scale is 20 and values in white regions are greater than 20. Incident field $E_{\text {in }}$ is polarized alone the $x$ axis.

relevant to excitation of the $\mathrm{TM}_{2}^{S}$ and $\mathrm{TM}_{3}^{S}$ modes, which are quadrupole and hexapole SP resonances on the outer surface of single metallic nanoshells, respectively. Nevertheless, due to SP interactions among spheres the two peaks have obvious energy shift and field distribution deformation with respect to the individual sphere SP modes.

The resonance at $\lambda_{4}$ has a character different from the transmission resonances at $\lambda_{1}$ and $\lambda_{2}$ discussed above. For example, the enhanced field is no longer confined within the nanogaps between spheres but extends into the surrounding medium (i.e., air) from the particle surface with multiple nodes and a longer decay length. The unique field distribution associated with a huge field enhancement (with a maximum of $\sim 300$ ) suggests that a kind of propagating surface mode may be excited at this resonance. Since the metal nanospheres are densely packed, SP interactions on adjacent spheres are strong enough for the SP to hop from sphere to sphere to form a propagating surface wave. ${ }^{18}$ As we will show later, this mode corresponding to peak 4 follows approximately the dispersion characteristics of a Bragg-type SPP. ${ }^{1,2}$

Note that peak 4 will become narrow in bandwidth upon increasing sphere separations, and will eventually disappear when the separation is too large because in this situation, plasmon hopping between spheres via near-field coupling is suppressed. To demonstrate this, Fig. 3 shows the normalincidence transmission spectra of a series of hollow metallic nanoshell arrays with increasing separation from 20 to $50 \mathrm{~nm}$ 


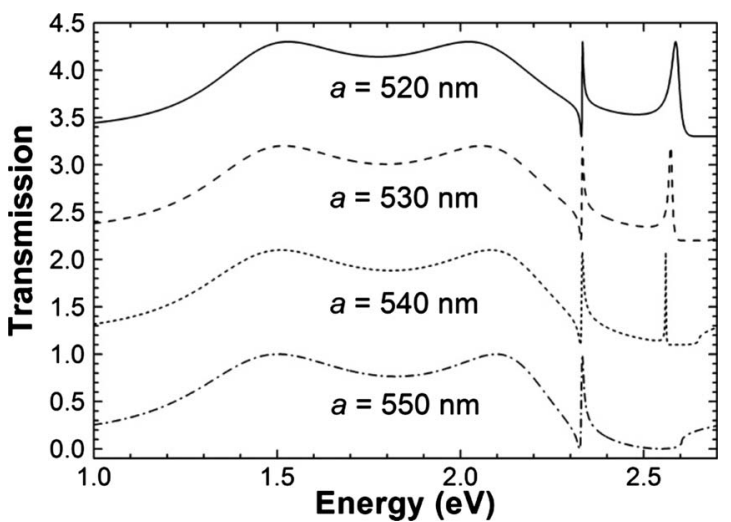

FIG. 3. Normal-incidence transmission spectra of 2D HCP arrays of hollow metallic nanoshells for different array periods $a$ $=520,530,540$, and $550 \mathrm{~nm}$. Other structural parameters are the same as those used in Fig. 1. Individual spectra are vertically offset by 1.1 from one another for clarity.

in steps of $10 \mathrm{~nm}$. It is evident that peak 4 gets progressively narrower as the distance between metallic spheres increases, and finally vanishes when $a$ is increased to $550 \mathrm{~nm}$. In contrast, there is almost no change in peak 3 upon increasing $a$, due to the highly localized nature of the $\mathrm{TM}_{1}^{V}$ mode. Peaks 1 and 2 show only a slight shift in position for this small change in $a$ as it could lead to a slight modification in the intersphere coupling of the $\mathrm{TM}_{2}^{S}$ and $\mathrm{TM}_{3}^{S}$ plasmon modes.

Now we turn to study the dispersion properties of the different resonant modes, by calculating the transmittance under off-normal incidence of light. For this purpose, Fig. 4 plots the transmission coefficients of the HCP hollow nanoshell array as a function of incidence angle $\theta$ and photon energy at two typical azimuth angles $\varphi=0^{\circ}$ and $30^{\circ}$ for both $p$ and $s$ polarizations. It is seen that the $\mathrm{TM}_{1}^{V}$ voidlike mode has an energy which is independent of the incident direction and polarization of light because of its highly localized nature and the high symmetry of the spherical cavities. It appears as a nondispersive bright horizontal line located at 2.33 $\mathrm{eV}$ in all four intensity maps in Fig. 4.

As is clearly seen in Fig. 4, peak 4 splits in energy into several modes at oblique incidence whose positions are all angle-dependent, suggesting a delocalization nature of these modes. It is interesting to see whether some of spectral features can be identified with Bragg-scattered SPP modes. To do this, yellow dashed lines are overlaid in Fig. 4 that describe the dispersion relations calculated by matching the momentum of a SPP on a smooth metal-dielectric interface with the reciprocal vector $G_{m n}$ of a 2D hexagonal lattice: $E_{S P P}=\hbar c\left[\left(\varepsilon_{2}+1\right) / \varepsilon_{2}\right]^{1 / 2}\left|G_{m n}+k_{x y}\right|$, where $c$ is light speed in vacuum, and $m$ and $n$ are integers. The analytical expression predicts well the position of peak 4 at normal incidence of light. Interestingly, some of the peaks and dips do follow the dispersion lines to a good approximation after splitting [see, for example, the lines labeled as $G_{-10} G_{-11}$ in Fig. 4(a) and $G_{01} G_{0-1}$ in Fig. 4(c)], further supporting the picture that this transmission peak is a propagating SPPs-mediated resonance. However, many dispersive features have no correspondence to the planar interface SPP dispersion folded by Bragg scattering (yellow lines). This is not surprising as the
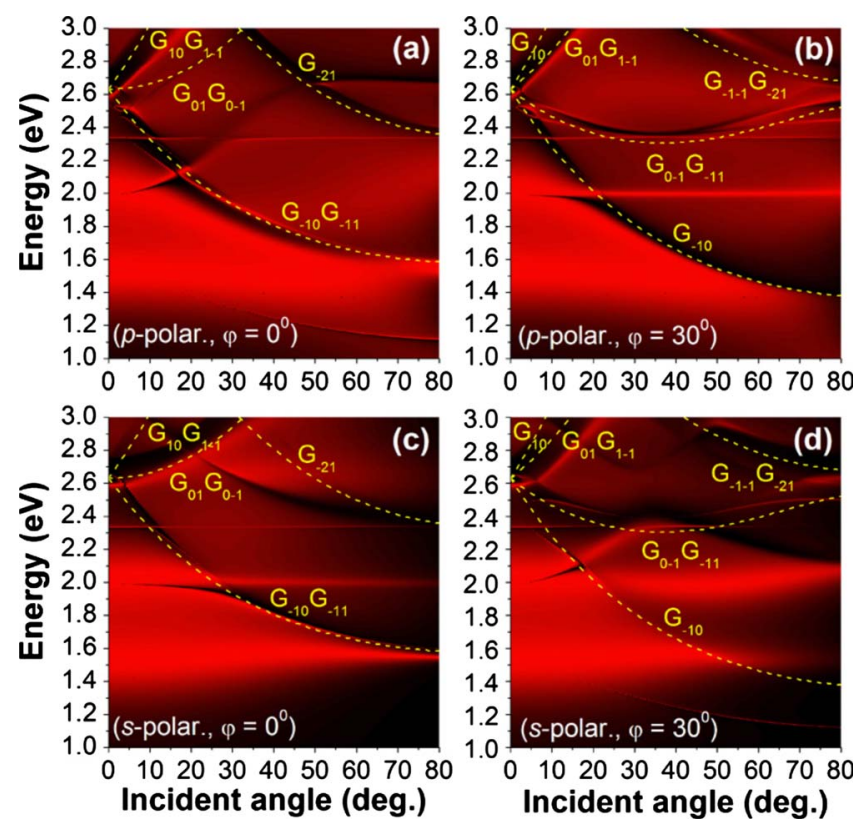

FIG. 4. (Color online) Dispersion maps for a 2D HCP array of metallic nanoshells at two azimuth angles $\varphi=0^{\circ}$ and $30^{\circ}$ for both $p$ and $s$ polarizations of incident light. Structural parameters are the same as those used in Fig. 1. The linear color scale for transmittance is set from black (minimum $T=0$ ) to red (maximum $T=1$ ). Overlaid yellow dashed lines represent the dispersion relations of SPPs modes associated with reciprocal vectors $G_{m n}$ of the 2D lattice.

plasmonic bands formed by the hopping of SPs on disjointed spheres should be different from SPPs of planar metal films interrupted by subwavelength scattering objects. ${ }^{1,2}$

For the spherelike modes contributing to two broad transmission bands in the low-energy region at normal incidence, the mode at $\omega_{1}=1.53 \mathrm{eV}$ becomes narrower in bandwidth as $\theta$ is increased (especially for $s$ polarization), since the mode is in essence localized at the nanogaps between adjacent metallic nanoshells. The resonance centered at $\omega_{2}=2.02 \mathrm{eV}$ gradually merges into the lowest dispersion line of the Bragg-type mode after $\theta \sim 10^{\circ}$, due to its interaction with SPP mode [see Fig. 4(a)-4(c)] but sustains as a wide-band peak with small dispersion when the coupling becomes weak [see Fig. 4(d)].

In the dispersion maps of Fig. 4 we found a highly localized transmission resonance. This resonant mode shows up as a horizontal, relatively narrow transmission band centered at about $2.0 \mathrm{eV}$ in Figs. 4(b) and 4(c). To get more insight into this narrow-band mode, we plot in Fig. 5 the corresponding electric field distributions in the six planes located at different distances from the $x y$ plane $(z=0)$ when the MD structure is illuminated with a $p$-polarized light with an energy $\omega=2.0 \mathrm{eV}$ under $\theta=30^{\circ}$ and $\varphi=30^{\circ}$. Clearly, the excited-field pattern is quite different from that of the modes excited at normal incidence of light (Fig. 2, right column). As is shown in the four planes $z= \pm 0.3 a$ and $z= \pm 0.4 a$ which are symmetrically around $z=0$, the fields are mainly concentrated in the pores of the lattice. This field localization in the pore region can still be resolved even at distances $z$ $= \pm 0.5 a$, although the maximum intensity is much reduced. 


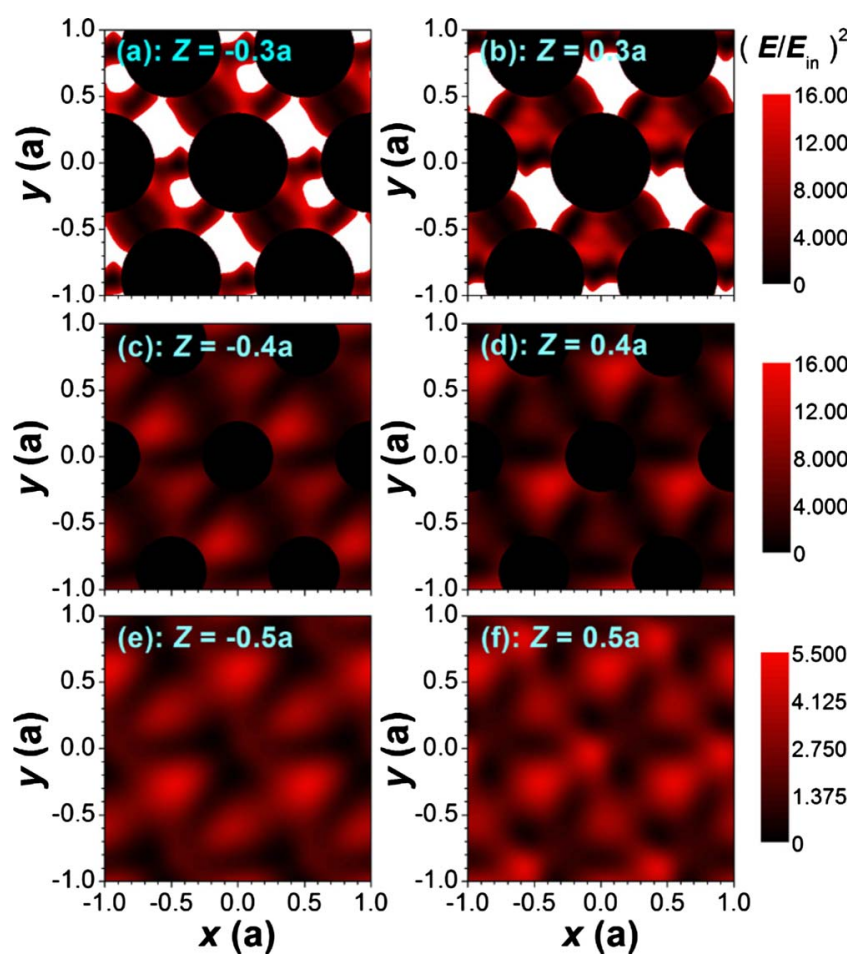

FIG. 5. (Color online) Normalized electric field intensity $\left(E / E_{i n}\right)^{2}$ in the six planes of $z= \pm 0.3 a$ (top row), $z= \pm 0.4 a$ (middle row), and $z= \pm 0.5 a$ (bottom row) for a $p$-polarized light with energy $\omega=2.0 \mathrm{eV}$ incident at $\theta=30^{\circ}$ and $\varphi=30^{\circ}$.

Based on the field distribution characteristics, we could refer to this resonant mode as a porelike mode. Strong localization of this mode also occurs in Fig. 4(c) at exactly the same energy, but becomes weak in Fig. 4(d) and eventually vanishes in Fig. 4(a). The reason for this is unclear yet, but may be related to the anisotropy of the pore shape, which leads to different coupling strengths with the far field. Since the porelike mode is a hybridization of the plasmons on individual nanoshells, it contains an admixture of nanoshell plasmons of different angular momenta.

The resonance position of the porelike mode could be tuned by varying the lattice period $a$ and thus the pore size for arrays of metallic nanoshells with fixed structural parameters. Figure 6 shows the same dispersion maps as in Fig. 4 for the same metallic nanoshells arranged in a HCP lattice, but with a period of $501 \mathrm{~nm}$ thus with an intersphere separation of only $1 \mathrm{~nm}$. In this nearly touching case, the two spherelike modes get further overlapped and the porelike mode almost moves out of the bandwidth of the spherelike modes at small angles of incidence, as shown in Figs. 6(b) and $6(\mathrm{c})$. Such a decrease in period $a$ also results in a redshift of the porelike mode, possibly because part of its field is squeezed out of the pores, leading to an increase in the mode volume. Note that the porelike mode exhibits a stronger localization in Figs. 6(a) and 6(d) than in Figs. 4(a) and 4(d), appearing as a flat narrow transmission band centered at $1.8 \mathrm{eV}$ from about $\theta=10^{\circ}$ to $35^{\circ}$. In addition, the yellow dispersion lines of Bragg-scattered SPPs move upward as a whole as compared with those in Fig. 4, since the SPPs energies will increase when $a$ is decreased. Although these dis-
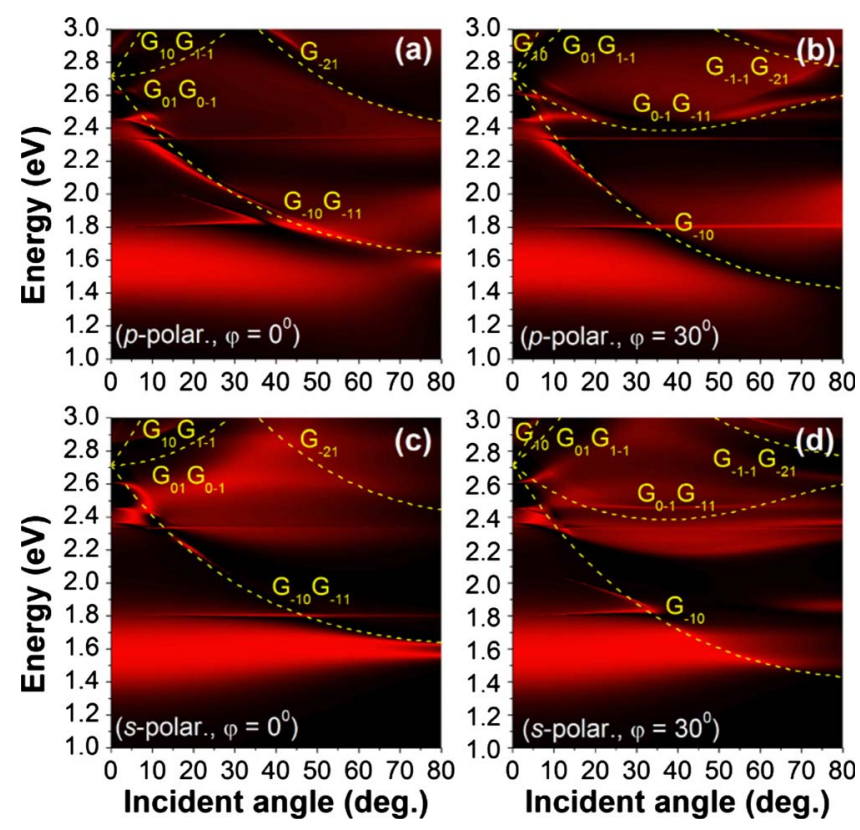

FIG. 6. (Color online) The same as Fig. 4 but for the lattice period $a=501 \mathrm{~nm}$.

persion lines have many deviations from the practical transmission resonance positions, they still show an agreement with the spectral features for some resonances revealed numerically in Fig. 6 to a good approximation. We mention that the energy of the voidlike mode $\mathrm{TM}_{1}^{V}$ is identical to that in Fig. 4 because of its highly localized nature.

Next we discuss the effects of interactions between different resonant modes on the transmittance. We first study the coupling between the porelike and spherelike modes. In Fig. 4 a noticeable gap around $2.0 \mathrm{eV}$ is observed from about $\theta$ $=5^{\circ}$ to $15^{\circ}$ in the wide transmission band of spherelike modes for all polarization and azimuth angles. It is clear that this transmission gap arises from the anticrossing of the two modes and this effect makes the spherelike mode centered at $\omega_{2}=2.02 \mathrm{eV}$ be cut into two parts. Such a gap also occurs in Fig. 6 at about $1.8 \mathrm{eV}$ within the region of smaller angles of incidence for a $1 \mathrm{~nm}$ intersphere separation. Note that we do not observe the gaps at normal incidence in Figs. 4 and 6. This could be possibly because the porelike mode is excitable only at off-normal incidence of light. Very recently Tserkezis et al. have pointed that some hybrid plasmon modes in 2D square arrays of metallic nanoshells are inactive at normal incidence, and only could be excited at oblique incidence. $^{31}$

To observe the effect as a result of coupling between wide-band spherelike and narrow-band voidlike modes, the relative permittivity $\varepsilon_{1}$ of the dielectric core is increased from unity to tune the voidlike modes into the lower-energy regime where the spherelike modes are located. Figure 7 shows the calculated transmission spectra of the MD nanostructures at normal incidence when $\varepsilon_{1}$ is varied from 1.0 to 3.0 in steps of 0.5 . It is seen that although the increase in the dielectric permittivity within the metallic nanoshells does not bring about a large variation in the overall transmission, a sharp zero-transmittance dip is always created as the $\mathrm{TM}_{1}^{V}$ 


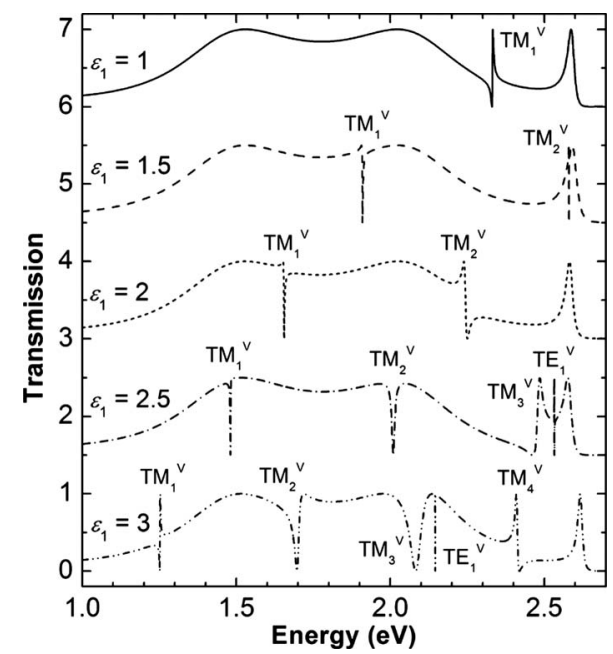

FIG. 7. Normal-incidence transmission spectra of 2D HCP arrays of metallic nanoshells. The dielectric core within the perfect metallic nanoshells takes different values of relative permittivity $\varepsilon_{1}=1.0,1.5,2.0,2.5$, and 3.0. Other structural parameters are the same as those used in Fig. 1. Individual spectra are vertically offset by 1.5 from one another for clarity.

voidlike mode is tuned across the broad band of the spherelike modes. This is the result of anticrossing (or spectral interference) between localized modes within and outside thin metallic nanoshells. Analogous interference phenomenon is commonly referred to as the Fano resonance in atomic physics. ${ }^{32}$ When the $\mathrm{TM}_{1}^{V}$ mode is tuned below the lower edge of the spherelike modes, a narrow-band transmission resonance is observed again for $\varepsilon_{1}=3$. Upon increasing $\varepsilon_{1}$, it is noted that the voidlike modes at higher energies will be simultaneously redshifted into the spectral range of interest, which can enable similar anticrossing effects, as is seen in Fig. 7.

In addition to the coupling between localized SP modes, by optimizing the structural parameters, our MD nanostructures can also allow interesting avoided crossing between propagating SPPs and various localized modes. Such a phenomenon has been discussed in other plasmonic structures. $^{20,29,33}$ For example, narrow zero-transmittance dips due to anticrossing between voidlike $\mathrm{TM}_{2}^{V}$ and $\mathrm{TE}_{1}^{V}$ modes and a SPP mode can be observed in Fig. 7, when $\varepsilon_{1}$ takes the values of 1.5 and 2.5 , respectively. Similar lowtransmission dips as a result of anticrossing between localized cavity modes and delocalized SPPs have been predicted in thin metal films buried with a monolayer dielectric sphere array by de Abajo et al. ${ }^{29}$ Similarly, the avoided crossing between highly dispersive SPPs and localized spherelike modes can also be seen in Figs. 4 and 6, which produces small gaps with a negligible transmittance.

We now consider the influence of light absorption by the shell metal. Figures 8(a)-8(c) shows, respectively, the normal-incidence transmission, reflection, and absorption spectra of a 2D HCP array of metallic nanoshells with different values of the loss parameter in the Drude model: $\hbar \tau^{-1}=0,0.05,0.1$, and 0.2 eV. In Fig. 8(a), it is seen that the salient features remain robust in the presence of absorption even when $\hbar \tau^{-1}$ is increased to $0.2 \mathrm{eV}$. The two relatively
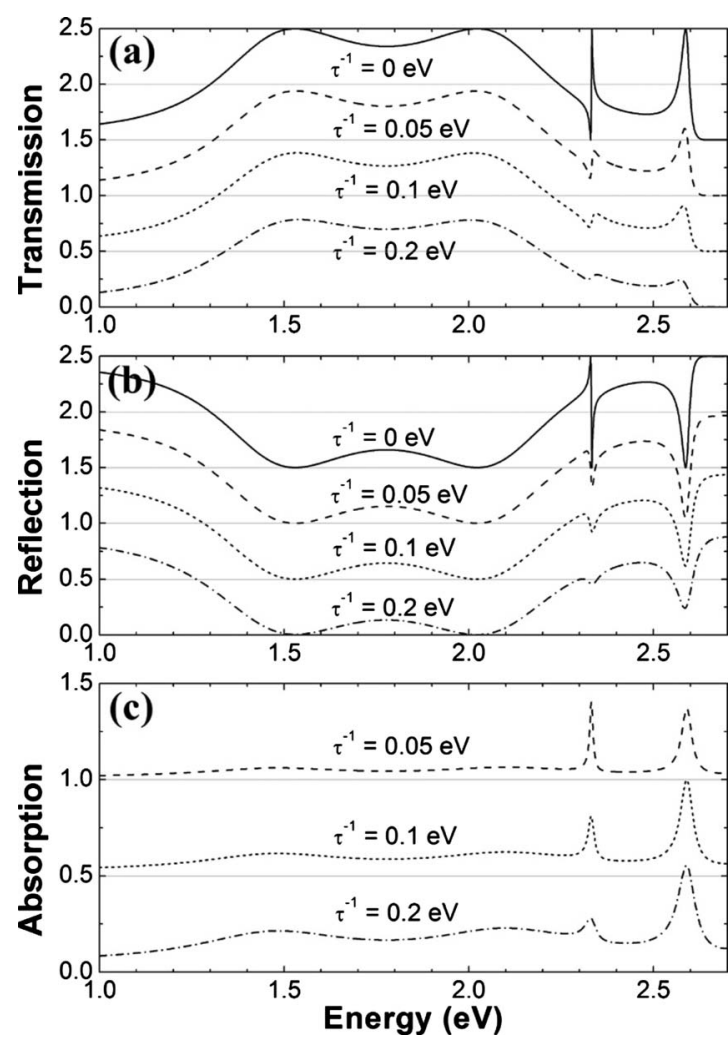

FIG. 8. Normal-incidence transmission (a), reflection (b), and absorption (c) spectra of a 2D HCP array of hollow metallic nanoshells for the shell metal to have different values of loss in the Drude model: $\hbar \tau^{-1}=0$ (perfect), 0.05, 0.1, and $0.2 \mathrm{eV}$. Other structural parameters are the same as those used in Fig. 1. Individual spectra are vertically offset by 0.5 from one another for clarity. Horizontal light-gray lines are guides to the eye.

narrow resonances, i.e., peaks 3 and 4 previously revealed in Fig. 1(b), however, are more affected by losses, which are smoothed out progressively as $\tau^{-1}$ increases, implying that their lifetime becomes more and more short. The reason is partly due to the larger Ohm loss in metal, as is clearly seen in Fig. 8(c), owing to the larger electric-field enhancements on the inner and outer surfaces of metallic nanoshells when voidlike and SPPs modes are excited, respectively.

On the other hand, for the two broad-band spherelike modes, the reflection at resonances is nearly equal to zero [less than 0.01, see Fig. 8(b)] for all values of $\tau^{-1}$, which indicates that the reduction in transmittance mainly arises from the Ohm loss in metal. For peaks 3 and 4, the larger the value of $\tau^{-1}$ is, the stronger is the reflection at the narrowband resonances. This means the radiative loss (reflection) will also contribute to the decrease in the transmittance.

Note that for the sharp resonance of the $\mathrm{TM}_{1}^{V}$ mode, the absorption peak decreases with increasing $\tau^{-1}$ [see Fig. 8(c)], unlike the behavior observed for the other three resonances. At first glance, this seems to be somewhat counterintuitive. But, this is not hard to be understood when realizing that light must penetrate into the metal shell in order to excite the voidlike Mie mode. Certainly, the increase in $\tau^{-1}$ will reduce the coupling strength of this mode, and thus the field enhancement on the inner surface of the metal shell. Conse- 
quently, the absorption peak amplitude will decrease when $\tau^{-1}$ is increased. When the metal is very lossy $\left(\tau^{-1}\right.$ is large $)$, the cavity mode cannot be excited and the corresponding absorption will vanish (not shown here).

Finally, it should be mentioned that the transmission peaks correspond to the reflectivity minima and absorbance maxima, a feature consistent with the involvement of SPs. ${ }^{34}$ The fields at the metal surface are enhanced when the incident light couples to a SP mode, thus aiding transmission. However, the SP mode is also damped by absorption in the metal so that enhanced absorption has the same conditions that give an enhanced transmission.

In conclusion, we have shown that light can tunnel through 2D dense HCP arrays of metal nanoshells via excitations of voidlike, spherelike Mie modes, Bragg-scattered SPPs, and porelike localized resonance modes. Voidlike Mie modes can be controlled by the size and dielectric constant of the dielectric core. The other three types of modes can be tuned by increasing the permittivity of surrounding medium or by scaling the array size. Moreover, transmission suppression phenomena due to anticrossing between different plasmon modes are revealed. Our study will be helpful to understand the physical mechanisms of anomalous light transmittance through a variety of metallic nanostructures. On the other hand, huge enhancement of electric fields at plasmon resonances suggests that the MD system can serve as a substrate for surface-enhanced spectroscopies. ${ }^{30}$

We thank C. T. Chan, L. Zhou, and J. Zi for helpful discussions and C. T. Chan for a critical reading of the manuscript. Acknowledgments for financial support are for C.T., Z.W. from the State Key Program for Basic Research of China and NSFC under grants No. 10425415, No. 10734010, No. 50771054, and No.10804044, for G.S. from NSFC under grant No. 10674157, and for S.Z. from NSFC under grant No. 10534020. *zlwang@nju.edu.cn

${ }^{1}$ T. W. Ebbesen, H. J. Lezec, H. F. Ghaemi, T. Thio, and P. A. Wolff, Nature (London) 391, 667 (1998); H. F. Ghaemi, T. Thio, D. E. Grupp, T. W. Ebbesen, and H. J. Lezec, Phys. Rev. B 58, 6779 (1998).

${ }^{2}$ J. A. Porto, F. J. García-Vidal, and J. B. Pendry, Phys. Rev. Lett. 83, 2845 (1999); L. Salomon, F. Grillot, A. V. Zayats, and F. de Fornel, ibid. 86, 1110 (2001); L. Martín-Moreno, F. J. GarcíaVidal, H. J. Lezec, K. M. Pellerin, T. Thio, J. B. Pendry, and T. W. Ebbesen, ibid. 86, 1114 (2001).

${ }^{3}$ M. M. J. Treacy, Phys. Rev. B 66, 195105 (2002); H. J. Lezec and T. Thio, Opt. Express 12, 3629 (2004).

${ }^{4}$ F. Yang and J. R. Sambles, Phys. Rev. Lett. 89, 063901 (2002); K. J. Klein Koerkamp, S. Enoch, F. B. Segerink, N. F. van Hulst, and L. Kuipers, ibid. 92, 183901 (2004); Z. Ruan and M. Qiu, ibid. 96, 233901 (2006); J. W. Lee, M. A. Seo, D. H. Kang, K. S. Khim, S. C. Jeoung, and D. S. Kim, ibid. 99, 137401 (2007).

${ }^{5}$ K. L. Kelly, E. Coronado, L. L. Zhao, and G. C. Schatz, J. Phys. Chem. B 107, 668 (2003).

${ }^{6}$ S. Nie and S. R. Emory, Science 275, 1102 (1997); H. Xu, E. J. Bjerneld, M. Käll, and L. Börjesson, Phys. Rev. Lett. 83, 4357 (1999).

${ }^{7}$ P. Mühlschlegel, H. J. Eisler, O. J. F. Martin, B. Hecht, and D. W. Pohl, Science 308, 1607 (2005).

${ }^{8}$ M. Righini, G. Volpe, C. Girard, D. Petrov, and R. Quidant, Phys. Rev. Lett. 100, 186804 (2008); A. N. Grigorenko, N. W. Roberts, M. R. Dickinson, and Y. Zhang, Nat. Photonics 2, 365 (2008).

${ }^{9}$ B. Lamprecht, G. Schider, R. T. Lechner, H. Ditlbacher, J. R. Krenn, A. Leitner, and F. R. Aussenegg, Phys. Rev. Lett. 84, 4721 (2000)

${ }^{10}$ E. M. Hicks, S. Zou, G. C. Schatz, K. G. Spears, R. P. Van Duyne, L. Gunnarsson, T. Rindzevicius, B. Kasemo, and M. Käll, Nano Lett. 5, 1065 (2005).

${ }^{11}$ K. T. Carron, W. Fluhr, M. Meier, A. Wokaun, and H. W. Lehmann, J. Opt. Soc. Am. B 3, 430 (1986).

${ }^{12}$ S. Zou, N. Janel, and G. C. Schatz, J. Chem. Phys. 120, 10871
(2004).

${ }^{13}$ V. A. Markel, J. Phys. B 38, L115 (2005).

${ }^{14}$ V. G. Kravets, F. Schedin, and A. N. Grigorenko, Phys. Rev. Lett. 101, 087403 (2008).

${ }^{15}$ B. Auguié and W. L. Barnes, Phys. Rev. Lett. 101, 143902 (2008).

${ }^{16}$ Z. Chen, P. Zhan, Z. L. Wang, J. H. Zhang, W. Y. Zhang, N. B. Ming, C. T. Chan, and P. Sheng, Adv. Mater. 16, 417 (2004).

${ }^{17}$ B. N. Khlebtsov, V. A. Khanadeyev, J. Ye, D. W. Mackowski, G. Borghs, and N. G. Khlebtsov, Phys. Rev. B 77, 035440 (2008); F. Le, D. W. Brandl, Y. A. Urzhumov, H. Wang, J. Kundu, N. J. Halas, J. Aizpurua, and P. Nordlander, ACS Nano 2, 707 (2008).

${ }^{18}$ S. A. Maier, P. G. Kik, H. A. Atwater, S. Meltzer, E. Harel, B. E. Koel, and Ari A. G. Requicha, Nature Mater. 2, 229 (2003).

${ }^{19}$ S. Coyle, M. C. Netti, J. J. Baumberg, M. A. Ghanem, P. R. Birkin, P. N. Bartlett, and D. M. Whittaker, Phys. Rev. Lett. 87, 176801 (2001).

${ }^{20}$ T. A. Kelf, Y. Sugawara, J. J. Baumberg, M. Abdelsalam, and P. N. Bartlett, Phys. Rev. Lett. 95, 116802 (2005); T. A. Kelf, Y. Sugawara, R. M. Cole, J. J. Baumberg, M. E. Abdelsalam, S. Cintra, S. Mahajan, A. E. Russell, and P. N. Bartlett, Phys. Rev. B 74, 245415 (2006).

${ }^{21}$ G. Sun and C. T. Chan, Phys. Rev. E 73, 036613 (2006).

${ }^{22}$ N. Stefanou, V. Yannopapas, and A. Modinos, Comput. Phys. Commun. 113, 49 (1998); 132, 189 (2000).

${ }^{23}$ V. Yannopapas, N. Stefanou, and A. Modinos, Phys. Rev. Lett. 86, 4811 (2001).

${ }^{24}$ Z. L. Wang, C. T. Chan, W. Y. Zhang, Z. Chen, N. B. Ming, and P. Sheng, Phys. Rev. E 67, 016612 (2003).

${ }^{25}$ W. Y. Zhang, X. Y. Lei, Z. L. Wang, D. G. Zheng, W. Y. Tam, C. T. Chan, and P. Sheng, Phys. Rev. Lett. 84, 2853 (2000); Z. L. Wang, C. T. Chan, W. Y. Zhang, N. B. Ming, and P. Sheng, Phys. Rev. B 64, 113108 (2001).

${ }^{26}$ M. I. Tribelsky and B. S. Luk'yanchuk, Phys. Rev. Lett. 97, 263902 (2006).

${ }^{27}$ C. F. Bohren and D. R. Huffman, Absorption and Scattering of Light by Small Particles (Wiley, New York, 1983). 
${ }^{28}$ E. Prodan, C. Radloff, N. J. Halas, and P. Nordlander, Science 302, 419 (2003).

${ }^{29}$ F. J. García de Abajo, G. Gómez-Santos, L. A. Blanco, A. G. Borisov, and S. V. Shabanov, Phys. Rev. Lett. 95, 067403 (2005).

${ }^{30}$ F. J. García-Vidal and J. B. Pendry, Phys. Rev. Lett. 77, 1163 (1996).
${ }^{31}$ C. Tserkezis, G. Gantzounis, and N. Stefanou, J. Phys.: Condens. Matter 20, 075232 (2008).

${ }^{32}$ U. Fano, Phys. Rev. 124, 1866 (1961).

${ }^{33}$ Y. J. Bao, R. W. Peng, D. J. Shu, M. Wang, X. Lu, J. Shao, W. Lu, and N. B. Ming, Phys. Rev. Lett. 101, 087401 (2008).

${ }^{34}$ W. L. Barnes, W. A. Murray, J. Dintinger, E. Devaux, and T. W. Ebbesen, Phys. Rev. Lett. 92, 107401 (2004). 